\title{
小麦籽粒钙元素含量的研究进展
}

\author{
刘玉秀 ${ }^{1} \quad$ 黄淑华 $^{2}$ 王景琳 $^{1}$ 张正茂 $^{1, *}$
}

${ }^{1}$ 西北农林科技大学农学院, 陕西杨凌 $712100 ;{ }^{2}$ 西北农林科技大学园艺学院, 陕西杨凌 712100

摘 要: 提高矿物质营养元素含量正在成为世界主要粮食作物的重要研究方向和育种目标。钲元素是人体必需的矿 物质元素, 在人类骨骼形成和新陈代谢中发挥着重要作用。全球约 35 亿人缺钙, 缺钻已成为影响人类健康的国际性 重大问题。主食是一种最优安全的矿物质元素补充途径。小麦是我国乃至全世界主要粮食作物, 是全球 $35 \%$ 珄\%人 口主要的食物来源, 是摄入钙的主要来源, 是矿物质元素生物强化的重要作物。通过遗传改良方法提高小麦籽粒钙元 素含量被认为是解决缺钻最经济、有效、可持续的措施, 目前已引起了国内外学者的高度关注。本文综述了近年来 小麦籽粒钙元素含量的研究进展, 主要包括籽粒钙含量的遗传差异、影响因素以及与相关性状关系、调控机理。此 外, 我们还提出了将来进行钙营养强化小麦研究的方向, 此研究内容为加快通过主粮实现有效补钙、倡导健康营养的 膳食模式、满足由 “量” 的需求向 “质” 的需求转变的粮食安全、改善国民健康状况以及减少因缺钙造成的经济损 失提供了解决方案。

关键词: 小麦; 籽粒钻含量; 钲营养强化

\section{Research advance on calcium content in wheat grains}

\author{
LIU Yu-Xiu ${ }^{1}$, HUANG Shu-Hua ${ }^{2}$, WANG Jing-Lin ${ }^{1}$, and ZHANG Zheng-Mao ${ }^{1, *}$ \\ ${ }^{1}$ College of Agronomy, Northwest A\&F University, Yangling 712100, Shaanxi, China; ${ }^{2}$ College of Horticulture, Northwest A\&F University, Yangling \\ 712100, Shaanxi, China
}

\begin{abstract}
Increasing the mineral content is becoming the important research direction and major target for crops breeding in the world. Calcium is an essential mineral element for human health and plays a pivotal role in skeletogenesis and metabolism. It is estimated that about 3.5 billion people was suffered from calcium deficiencies. Calcium deficiency has become a major international problem harming human health. The staple food is an optimal and safe way to mineral supplement. Wheat, one of the main food crops in China and even in the world, is the main source of food for $35 \%-40 \%$ of global population, a main source for human's calcium intake as well as an important crop of mineral element biofortification. Improving the calcium content in wheat grains through genetic improvement is considered to be the most economical, effective and sustainable measure to solve the calcium deficiency, which has aroused great concern from international scholars. This paper summarized the recent advances in the study of calcium content in wheat grains, mainly including the genetic variation, affecting factors, the relationship with related traits and regulation mechanism of calcium content in grain. Furthermore, we also put forward the direction of future research on calcium-fortified wheat, which provides solutions for accelerating the effective calcium supplementation through staple food, promoting the healthy and nutritious dietary pattern, ensuring the food security to meet the transition from "quantitative" to "qualitative" demands, improving people's health, and reducing economic losses caused by calcium deficiency.
\end{abstract}

Keywords: wheat; calcium content in grain; calcium fortification 
人群在非洲和亚洲, 缺钲已成为影响人类健康的世 界性重大问题 ${ }^{[2-3]}$ 。2012 年我国居民人均钙摄入量为 $366.1 \mathrm{mg} \mathrm{d}^{-1}$, 不到中国营养学会推荐钙摄入量的一 半, 且低于 2002 年 $388.8 \mathrm{mg}{ }^{[4]}$ 。缺钙可引起骨质疏 松症、骨关节病、小儿佝偻病等疾病 ${ }^{[1,5]}$, 其中仅缺 钙引起的骨质疏松性骨折, 美国和我国每年需花费 上百亿的医疗费用 ${ }^{[6]}$ 。另外, 饮食中低钙摄入量与肾 结石和结肠癌的风险增加有关 ${ }^{[7]}$ 。

人类借助富含钙的食物、保健品或药品等进行 补钙, 但依然存在投资大、覆盖面小和费用高等缺 陷, 不能根治钙营养不良的问题。主食是一种最优 安全的矿物质元素补充途径。在食品加工过程中添 加营养强化剂, 提高食品中钙含量, 如钙强化五浆、

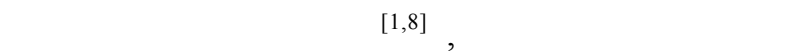
覆盖面不广。生物强化是一种基于农业的改善人体 营养的工具。生物强化是通过遗传改良方法提高小 麦籽粒的钙等矿物质元素含量, 是在小麦生长过程 中自然而然的增加, 且不影响作物的农艺性状和口 感特性 ${ }^{[9-11]}$ 。粮食作物的生物强化是一种低成本、可 持续和有效解决钙缺乏的方案 ${ }^{[12]}$ 。

小麦是分布最广的世界性粮食作物, 为人类提 供约 $20 \%$ 的蛋白质和 $21 \%$ 的食物热量, 是人体膳食 钙的主要来源。小麦作为全球 $35 \%$ $40 \%$ 人群赖以生 存的主要粮食之一, 是矿物质元素生物强化的重要 对象 ${ }^{[13-14]}$ 。小麦钙生物强化对提高小麦营养价值, 从而解决全球人口尤其是贫困地区人口由于钙缺乏 造成的健康问题具有重要现实意义。因此, 本文从 小麦籽粒钙元素含量的遗传差异、影响因素、其与 相关性状关系以及分子调控机理的角度, 综述其研 究进展, 为钙营养强化小麦种质资源创新、新品种选 育与富钙小麦食品研究开发利用提供一定的参考。

\section{1 小麦籽粒钙含量的测定方法}

钙含量测定比较常见的方法有原子吸收光谱法 $(\mathrm{AAS})^{[15-16]}$ 、原子吸收分光光度计分析法

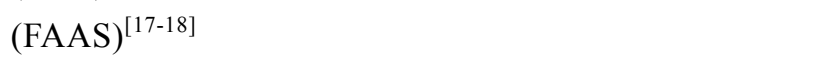
$(\mathrm{ICP}-\mathrm{AES})^{[19-20]}$ 和电感耦合等离子体质谱法 $(\mathrm{ICP}-\mathrm{MS})^{[21]}$ 等。AAS方法具有快速、准确、不干扰 等优点, 但需要繁琐的样品预处理过程 ${ }^{[15]}$ 。FAAS方 法, 样品的前处理是其分析结果的关键影响因素, 其中高温灰化法是最常用的前处理方法之一, 具有 消化完全、消化时间长和操作较复杂等特点; 而盐 酸浸提法具有操作简便、污染少和处理时间较短等
特点。以上方法均存在费用高、预处理过程繁杂、 易受到污染、难于对育种材料进行大规模分析和筛 选等缺点 ${ }^{[13,22]}$ 。相比以上方法, X-射线能谱仪法具有 快速、准确、不需化学试剂和提取等操作步骤等优 点, 适用于篮选富含必需矿质元素的育种材料或测 定籽粒各部位矿质元素(除 $\mathrm{H}$ 和 $\mathrm{He}$ 元素外)组成及分 布情况, 但只能测定元素的相对含量, 需标准样品 才能测定绝对含量 ${ }^{[23]}$ 。近年来逐渐得到科研工作者 关注的X射线苂光光谱法 $(X R F)$, 具有样品前处理准 备简便、多元素同时测定、重现性好、准确度高等

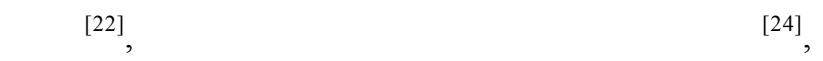
其中采用X-Supreme 8000 (Oxford Instrument plc, Abingdon, UK) 或 Bruker S2 Ranger (Bruker Corporation, Massachusetts, USA)仪器, 具有无损伤、低 成本、高通量、高效率等优点 ${ }^{[13]}$ 。钙元素不直接吸 收近红外光, 但在植物体内与有机物结合使得钙元 素可被近红外光谱技术(NIR)间接测定。利用NIR技 术对大米、烟叶中钙元素的含量进行了测定分析, 分别应用修正的偏最小二乘回归分析(MPLS)和最 小二乘支持向量机(LS-SVM)建立了预测模型 ${ }^{[25-26]}$, 可用于种质资源的快速测定和篮选。在试验和生产 中, 测定小麦籽粒钙含量应根据具体情况选用适宜 的测定方法。

\section{2 小麦籽粒钙含量的差异及影响因素}

\section{1 小麦基因型间籽粒钙含量的差异}

小麦籽粒钙含量受基因型、环境及两者互作的 显著影响, 但基因型效应远大于基因型与环境互作 效应 ${ }^{[27-30]}$ 。不同基因型小麦种质籽粒钲含量的变异 范围较宽, 基因型间存在显著差异。我国小麦微核 心种质库的 262 份种质籽粒钙含量变异范围为 290 976 mg kg-1 [19], 北方地区主推小麦品种为 $317 \sim 653 \mathrm{mg} \mathrm{kg}^{-1}{ }^{[28]}$, 黄淮麦区小麦骨干种质为 40.79 954.80 mg kg${ }^{-1}$ [31], 豫北地区主栽小麦品种为 $376.23 \sim 693.73 \mathrm{mg} \mathrm{kg}^{-1}{ }^{[32]}$, 长江中下游地区主推小 麦品种和部分国内外引进小麦品种籽粒钙含量介于 $242.66 \sim 620.51 \mathrm{mg} \mathrm{kg}^{-1}$ [16]。山农 $483 \times$ 川 35050 构建 的小麦重组自交系群体籽粒钙含量范围为 317 $737 \mathrm{mg} \mathrm{kg}^{-1}$ [33]。中国 15 个小麦主要种植区以及俄 罗斯等 7 个国家小麦种质籽粒钲含量分布范围为 $139.18 \sim 676.04 \mathrm{mg} \mathrm{kg}^{-1}$ [34]。欧洲小麦品种籽粒钙含 量变异范围为 $288.2 \sim 647.5 \mathrm{mg} \mathrm{kg}^{-1}$ [30], 葡萄牙小麦 种质为 $381 \sim 496 \mathrm{mg} \mathrm{kg}^{-1}[3]$, 美国小麦种质为 $242 \sim$ 
$765 \mathrm{mg} \mathrm{kg}^{-1}$ [29]，巴基斯坦小麦种质为 295 $455 \mathrm{mg} \mathrm{kg}^{-1}$ [35]。土耳其农家种籽粒钙含量为 340 $685 \mathrm{mg} \mathrm{kg}^{-1}$ [36], 其硬粒小麦品种为 $290 \sim 685 \mathrm{mg} \mathrm{kg}^{-1}[37]$ 。 山羊草属(Aegilops L. species)颖果钙含量高于普通 小麦籽粒，野生一粒小麦、二粒小麦和斯佩耳特小 麦籽粒钙含量高于栽培种品种, 是富集钙的潜在种 质资源 ${ }^{[38]}$ 。人工合成六倍体小麦籽粒钙含量低于面 包小麦品种 ${ }^{[39]}$, 变异范围为 $21.6 \sim 167.2 \mathrm{mg} \mathrm{kg}^{-1}$ [21]。 有芒小麦品种籽粒钙含量高于无芒小麦品种 ${ }^{[40]}$ 。彩 色小麦籽粒钙含量高于普通白粒小麦 ${ }^{[41]}$ 。通过测定 分析, 䇛选到了一些高钙含量小麦种质, 如徐麦 $856^{[28]}$ 、豫麦 $47^{[28]}$ 、郑麦 $9405^{[28]} 、 \mathrm{NP} 164^{[16]}$ 、地 方品种 $47^{[36]}$ 、Eyyubi ${ }^{[40]}$ 等, 可作为当地优良的高 钙含量种质资源材料供育种利用。然而, 如何进一 步评估和拓宽这类种质资源, 并借助遗传改良的 方法生产富含钙营养元素并有益于人类健康的粮 食, 这也成为作物科学和国际营养科学研究的一 个热点领域。

\section{2 小麦籽粒不同部位钙含量的差异}

小麦籽粒和糊粉层中均富含钙元素, 胚乳层中 钙含量比皮层和糊粉层低, 不同小麦品种籽粒各部 位的钙含量存在遗传性差异 ${ }^{[23,42]}$ 。钙含量在敖皮中 最高, 变异幅度最大; 籽粒中次之; 面粉中最低 ${ }^{[16]}$ 。 籽粒钙含量是面粉中的 1.11 2.31倍, 敖皮钙含量是 面粉中的 2.83 8.70 倍, 敖皮钙含量是籽粒的 $1.48 \sim 5.48$ 倍 ${ }^{[16]}$ 。

\section{3 农艺措施对小麦籽粒钙含量的影响}

在石灰性土壤中施用钙肥氯化钙可促进小麦干 物质形成, 增加穗数和籽粒产量, 显著增加整株钙 累积量, 但对籽粒钙含量无影响 ${ }^{[18]}$; 但在酸性淋溶 土中施用石灰，显著提高了小麦籽粒钙含量 ${ }^{[43]}$ 。无 论氮肥施用量如何, 接种巴西固氮螺菌可增加小麦 籽粒钙含量 ${ }^{[44]}$ 。施用腐殖酸 $(85 \%$ 腐殖酸)显著增加小 麦生物产量和籽粒产量; 随着腐殖酸施用量增加, 显著降低小麦籽粒钙含量 ${ }^{[45]}$ 。施用基于氨基酸的生 物刺激素(AminoPrim和AminoHort)有助于提高小麦 籽粒钙含量 ${ }^{[46]}$ 。小麦与绿豆、大豆、秋豆轮作, 其 籽粒钙含量增加; 随着施氮量增加, 小麦籽粒钙含 量增加 ${ }^{[47-48]}$ 。然而, 也有研究发现小麦等前茬作物 对冬小麦籽粒钙含量无明显影响, 施氮肥对籽粒钙

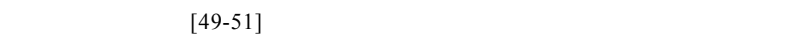
小麦籽粒钲含量 ${ }^{[52]}$ 。土壤施用锌肥显著提高了小麦 籽粒钙含量 ${ }^{[53]}$, 但喷施叶面锌肥 $\left(\mathrm{ZnSO}_{4} \cdot 7 \mathrm{H}_{2} \mathrm{O}\right.$,
$0.3 \%, \mathrm{w} / \mathrm{v})$ 降低了小麦籽粒钙含量 ${ }^{[54]}$ 。剪叶遮光可提 高小麦籽粒钙含量, 但降低了单穗钙产量 ${ }^{[55]}$ 。与传 统栽培方法相比，地膜覆盖、秸秆还田和种植绿肥 降低了旱地小麦籽粒钙含量 ${ }^{[56]}$; 与施氮量较高的常 规栽培相比, 少耕(覆盖耕作和免耕)和降低施氮量 对小麦籽粒钙含量增加效应更好 ${ }^{[57]}$ 。有机栽培管理, 尤其是不施可溶性肥料, 并没有引起小麦籽粒钙含 量的急剧增加 ${ }^{[58]}$ 。此外, 集约化种植制度提高了小 麦产量, 但同时降低了其营养品质, 籽粒养分钙含 量低 ${ }^{[59]}$ 。

\section{3 小麦籽粒钙含量与其他性状的关系}

\section{1 小麦籽粒钙含量与主要农艺性状的关系}

由于过去的小麦育种目标过分集中在产量和抗 逆性改良, 忽视其籽粒矿物质元素含量, 在一定程 度上导致育成的小麦品种籽粒矿物质含量较低 ${ }^{[3]}$ 。 小麦籽粒钲含量与千粒重、产量均呈显著负相关 ${ }^{[29]}$, 但也有研究发现小麦籽粒钙含量与千粒重呈显著正 相关 ${ }^{[37]}$ 。幼苗发育的第一阶段取决于种子中矿物质 元素的含量 ${ }^{[60]}$ 。二价钙离子 $\left(\mathrm{Ca}^{2+}\right)$ 调节和控制与光合 作用相关的气孔运动, 加速糖分运输, 增强光合效 率 $^{[61]}$ 。

3.2 小麦籽粒钙含量与品质和其他矿质元素的 关系

测定不同小麦品种(系)籽粒钙含量和品质相关性 状, 发现籽粒钙含量与粗蛋白含量、湿面筋和沉降值 之间呈正相关, 但无显著性 ${ }^{[20]}$ 。小麦籽粒钙含量与籽 粒蛋白质含量、锌含量、铁含量、硫含量、磷含量、 镁含量和锰含量呈显著正相关 ${ }^{[21,29,37-38,62-63]}$ 。

\section{3 小麦籽粒钙含量与其生物有效性影响因子} 的关系

禾谷类作物(小麦、水稻等)籽粒中含有一些影响 钙元素生物有效性的抗营养因子，它们不仅能与钙 形成螯合物，而且通过调控机制能调节钙的吸收、 转运以及在籽粒中的重新分配, 其中植酸是最主要 的抗营养因子，降低了钙的生物有效利用率 ${ }^{[64-65] 。}$ 小麦籽粒中钙主要以植酸盐螯合物形式存在, 在自 然界中无法自行降解, 只能通过植酸酶分解为低磷 肌醇衍生物和无机磷酸盐，才能被人体加以有效利 用 ${ }^{[13,66]}$ 。植酸在作物抵抗生物和非生物胁迫中起着 重要作用, 其含量在籽粒中保持在一定水平 ${ }^{[67]}$ 。因 此, 提高植酸酶活性可能是提高钙吸收利用率、解 决钙含量缺乏问题的重要途径。 


\section{4 小麦籽粒钙含量与面粉钙含量的关系}

小麦籽粒钙含量高, 面粉钙含量也高, 二者呈 极显著正相关 ${ }^{[16]}$, 说明从籽粒钙含量高的小麦种质 材料中有可能选育出面粉钙含量高的小麦品种。小 麦磨粉即去除麦敖制成精粉。小麦粉的加工精度越 高，所含的敖皮和胚芽越少，营养价值越低。利用生 产线上取得的各系统小麦粉配制成不同出粉率的面 粉，发现当出粉率为 $35 \%$ 70\% 时，面粉中钙含量保 持一个较低且稳定的含量; 但当出粉率高于 $80 \%$ 时, 其钙含量明显升高 ${ }^{[68]}$ 。利用布勒试验磨粉机制备面 粉, 随着出粉率的提高 $(66 \% \sim 78 \%)$, 小麦面粉钙含 量无显著变化 ${ }^{[69]}$ 。因此, 在不影响面粉食用品质和 加工品质的前提下, 适量降低面粉的精度, 提高面 粉出粉率，进而提高面粉中钙营养元素含量。

\section{4 小麦籽粒钙含量调控的机制研究}

\section{1 小麦籽粒钙含量积累的生理基础与遗传机 制研究}

高钙型植物具有较高的钙富集能力, 其地上部 分在低钙含量土壤中可维持较高的钙含量; 低钲型 植物反之亦然 ${ }^{[0]}$ 。蒸腾作用、导管壁上的阳离子吸 附及组织水势的变化影响植物体内钙的运输和分 配。尽管在细胞内钙转运研究取得了相当大的进展, 但迄今为止, 籽粒中高钙含量积累的分子基础研究 报道很少 ${ }^{[61]}$ 。小麦籽粒钙含量主要取决于根系吸收、 体内运输和再分配以及在种子中转移和积累等过 程 ${ }^{[71]}$ 。这些过程中的每一个都很可能受到多个基因 控制，使得钙在籽粒中的积累是一个复杂的多基因 现象。随着灌浆进程的推进, 小麦籽粒钙含量和钙 累积速率逐渐降低, 但籽粒钙总量逐渐提高; 叶片 和叶鞘钙不能向籽粒转运, 籽粒钙主要来自于根系 的转运 ${ }^{[72]}$ 。钙在韧皮部迁移水平显著影响其在籽粒 中的含量和位置, 而钙在韧皮部移动性差, 不易到 达籽粒胚乳，部分残留在小麦籽粒种皮上 ${ }^{[53]}$ 。到目 前为止, 对小麦籽粒中钙含量转运、积累的生理基 础与遗传机制还不是很清楚。

\section{2 小麦籽粒钙含量基因定位研究}

近十年来, 随着营养基因组学的发展, 营养生 物学科学得到了广泛的发展和进步。在营养隐形饥 饿日益严重的时代，必须利用谷物的营养特性来开 发具有新营养价值的谷物 ${ }^{[59]}$ 。从目前育种目标来看, 利用常规育种策略已培育出许多具有多种营养丰富 特性的潜在品种。然而, 小麦传统育种技术主要通
过表型性状对基因型进行间接选择，需要丰富的育 种经验和较长时间; 同时籽粒钙含量性状受多因素 影响, 选择效率低。基因组学辅助育种就为营养生 物学研究开辟一条新的途径。

数量性状基因座(QTL)分析是解析小麦矿物质 含量性状的有效方法, 广泛应用于作物数量性状遗 传研究。利用QTL分析能有效检测与小麦籽粒钙含 量的相关基因位点，从而提高富钙小麦育种的效 率。目前, 关于小麦籽粒钙含量的基因定位研究较 少。Peleg等 ${ }^{[73]}$ 通过连锁分析定位到 9 个控制籽粒钙 含量的QTL，位于 $1 \mathrm{~A} 、 4 \mathrm{~A} 、 6 \mathrm{~A} 、 2 \mathrm{~B} 、 4 \mathrm{~B} 、 5 \mathrm{~B} 、 6 \mathrm{~B}$ 和 $7 \mathrm{~B}$ 染色体(表1), 解释表型变异 $1.0 \%$ 21.0\%; 除了 4B染色体，位于其他染色体上的QTL均在所有环境 中检测到; 6B染色体上的QTL位点同时与钲、锌和铁 含量相关; 7B染色体上的QTL位点同时与钙、籽粒蛋 白质含量、钾和锰含量相关，说明这些QTL同时可用 于改良籽粒钙、锌、铁、钾和锰含量。Shi等 ${ }^{[74]}$ 在 $1 \mathrm{~A}$ 、 $2 \mathrm{~A} 、 7 \mathrm{~B}$ 和 $2 \mathrm{D}$ 染色体上定位到 4 个控制籽粒钙含量 QTL (表1), 解释表型变异 $5.8 \%$ 14.0\%; 在 $1 \mathrm{~A}$ 染色 体上检测到的QTL，其遗传距离位于10.1 10.9 cM 之间, 这与Peleg 等 ${ }^{[73]}$ 报道的QTL $(5.0 \sim 58.7 \mathrm{cM})$ 的异 同还有待于进一步验证; 在7B染色体上检测到的 QTL (0 9.7 cM) 与Peleg等 ${ }^{[73]}$ 报道的遗传距离位于 15.2 30.8 cM之间的QTL不同。Alomari等 ${ }^{[30]}$ 以 353 份欧洲小麦品种为材料，对籽粒钙含量进行全基因 组关联分析，共发现 485 个与籽粒钙含量显著关联 的单核苷酸多态性(SNP)标记，分布在除 $3 \mathrm{D} 、 4 \mathrm{~B}$ 和 $4 \mathrm{D}$ 以外的所有染色体上，其中在 2 个环境和BLUE值 (阈值为 $-\log _{10}(P$-value $\geq 3)$ ) 中均与籽粒钙含量显著 关联的标记位于 $2 \mathrm{~A} 、 3 \mathrm{~A} 、 5 \mathrm{~A} 、 6 \mathrm{~A} 、 5 \mathrm{~B}$ 和 $5 \mathrm{D}$ 染色体 上(表 1$)$; 在这 6 条染色体上与籽粒钙含量显著相关 的SNP位点附近找到了 41 个与钙吸收或转运相关的 候选基因。植物钙转运蛋白已被证实了可增加胡夢 卜等可食根的钙含量 ${ }^{[75]}$ 。在 $2 \mathrm{~A}$ 和 $6 \mathrm{~A}$ 染色体上检测的 位点与Shi等 ${ }^{[74]}$ 和Peleg等 ${ }^{[73]}$ 报道的不同。Bhatta等 ${ }^{[21]}$ 通过对 123 份人工合成六倍体小麦种质的籽粒钙含 量进行全基因关联分析，在 $3 \mathrm{~A} 、 6 \mathrm{~A} 、 7 \mathrm{~A} 、 1 \mathrm{~B} 、 2 \mathrm{~B}$ 、 $3 \mathrm{~B} 、 6 \mathrm{~B} 、 2 \mathrm{D}$ 和 $3 \mathrm{D}$ 染色体上发现了 15 个与籽粒钙含 量显著关联的 SNP 标记 (表1), 解释表型变异 $2.7 \%$ 21.5\%。由于所用群体(RILs、DHs或自然群体)、 标记不同(AFLP、SSR、DArTs、90K)或准确位置信 息缺乏或IWGSC RefSeq v1.0以外的其他版本参考 小麦基因组使用，在 $3 \mathrm{~A}$ 染色体上定位的QTL与 


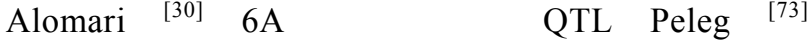
和Alomari等 ${ }^{[30]}$ 以及在 $2 B 、 6 B$ 染色体上定位的QTL 与Peleg等 ${ }^{[73]}$ 的异同还有待于进一步验证。Shen等 ${ }^{[63]}$ 在RILs群体中发现了位于4B染色体上调控钾、钙和 镁含量的QTL位点，但没有定位到控制籽粒钙含量 的QTL。以上研究仅限于初步QTL定位水平，尚末有 精细定位的研究结果, 也不清楚调控小麦籽粒钙含 量的基因是什么。钲依赖性蛋白激酶(CDPKs)作为植 物细胞中钲浓度变化的关键传感器，在小麦中鉴定
出 20 个 $C D P K$ 基因 ${ }^{[76]}$ 。近年来，在拟南芥、穇子 (finger millet)和水稻上有关籽粒钙含量的研究较多, 不仅开发了用于检测籽粒钙含量的EST-SSR标记和 SSR标记 ${ }^{[77-78]}$; 同时也发现了一些调控籽粒钙含量 基因，如 $C A X^{[61,79-80]}$ 和 $T P C 、 C a M K 、 E c C I P K$ 基因家 族 ${ }^{[59]}$ 。相对于籽粒钙含量，小麦籽粒中铁、锌、硒 元素基因定位的研究进展较快, 不仅QTL定位研究 较多 ${ }^{[11]}$, 而且还成功克隆到一个提高籽粒蛋白质、 铁和锌含量而对产量无影响的基因 $G P C-B 1^{[81]}$ 。

表 1 小麦籽粒钙含量 QTL 的汇总

Table 1 Summary of QTL detected for calcium content in wheat grains

\begin{tabular}{|c|c|c|c|c|}
\hline $\begin{array}{c}\text { 染色体 } \\
\text { Chr. }\end{array}$ & $\begin{array}{c}\text { 位置 } \\
\text { Position (cM or bp) }\end{array}$ & $\begin{array}{c}\text { 标记名称 } \\
\text { Molecular marker or interval marker }\end{array}$ & $\begin{array}{c}\text { 群体 } \\
\text { Population }\end{array}$ & $\begin{array}{l}\text { 参考文献 } \\
\text { References }\end{array}$ \\
\hline $1 \mathrm{~A}$ & $31.7 \pm 26.7$ & gwm3083 & RILs 群体 RILs population & Peleg et al. ${ }^{[73]}$ \\
\hline $4 \mathrm{~A}$ & $28.7 \pm 2.4$ & gwm610 & & \\
\hline $6 \mathrm{~A}$ & $106.9 \pm 20.2$ & wPt-0139 & & \\
\hline $2 \mathrm{~B}$ & $86.5 \pm 6.9$ & wPt-6576 & & \\
\hline 4B & $88.1 \pm 9.7$ & wPt-9393 & & \\
\hline $5 \mathrm{~B}$ & $54.2 \pm 5.9$ & gwm371 & & \\
\hline $6 \mathrm{~B}$ & $22.6 \pm 28.1$ & wPt-11506 & & \\
\hline $6 \mathrm{~B}$ & $145.4 \pm 20.7$ & gwm219 & & \\
\hline $7 \mathrm{~B}$ & $23.0 \pm 7.8$ & gwm 263 & & \\
\hline $1 \mathrm{~A}$ & $10.1-10.9$ & P3156.2-WMC59 & DHs 群体 DHs population & Shi et al. ${ }^{[74]}$ \\
\hline $2 \mathrm{~A}$ & $15.7-16.6$ & WMC27.2-P5166.2 & & \\
\hline 7B & $0-9.7$ & P1123.2-Xgwm611 & & \\
\hline $2 \mathrm{D}$ & $1.5-20.8$ & WMC41-WMC170 & & \\
\hline $2 \mathrm{~A}^{\mathrm{a}}$ & 64.3 & $\begin{array}{l}\text { RAC875_c24517_558, Kukri_c40035_258, } \\
\text { AX-94881950, AX-94850365, AX-94560505, } \\
\text { AX-94544896, AX-94404038 }\end{array}$ & 自然群体 Natural population & Alomari et al..$^{[30]}$ \\
\hline $2 \mathrm{~A}^{\mathrm{a}}$ & 66.6 & $\begin{array}{l}\text { BS00049644_51, AX-95169653, AX-94940052, } \\
\text { AX-94536561 }\end{array}$ & & \\
\hline $3 \mathrm{~A}^{\mathrm{a}}$ & 109 & RFL_Contig1175_354 & & \\
\hline $5 \mathrm{~A}^{\mathrm{a}}$ & 117.7 & wsnp_Ex_c20899_30011827, AX-95077733 & & \\
\hline $5 \mathrm{~A}^{\mathrm{a}}$ & 115.5 & RAC875_c8642_231 & & \\
\hline $6 \mathrm{~A}^{\mathrm{a}}$ & 37.3 & $\begin{array}{l}\text { wsnp_Ex_c17575_26300030, } \\
\text { wsnp_Ex_c17575_26299925, Tdu- } \\
\text { rum_contig62141_496, } \\
\text { Kukri_rep_c104648_439, Kukri_c35661_63, } \\
\text { AX-94415776 }\end{array}$ & & \\
\hline $5 \mathrm{~B}^{\mathrm{a}}$ & 75.5 & GENE-0168_7 & & \\
\hline $5 \mathrm{~B}^{\mathrm{a}}$ & 78.7 & RAC875_c30011_426, BS00062731_51 & & \\
\hline $5 \mathrm{~B}^{\mathrm{a}}$ & 100.9 & AX-94547820, AX-94452355 & & \\
\hline $5 \mathrm{~B}^{\mathrm{a}}$ & 101.7 & AX-94541836 & & \\
\hline $5 \mathrm{~B}^{\mathrm{a}}$ & 103.2 & AX-94644169 & & \\
\hline $5 \mathrm{~B}^{\mathrm{a}}$ & 149.8 & snp_CAP8_c1210_739429, CAP7_c5481_96 & & \\
\hline $5 \mathrm{D}^{\mathrm{a}}$ & 167 & Jagger_c8037_96 & & \\
\hline
\end{tabular}




\begin{tabular}{cclc}
\hline $\begin{array}{c}\text { 染色体 } \\
\text { Chr. }\end{array}$ & $\begin{array}{c}\text { 位置 } \\
\text { Position (cM or bp) }\end{array}$ & \multicolumn{1}{c}{ Molecular marker or interval marker } & \multicolumn{1}{c}{$\begin{array}{c}\text { 标记名称体 } \\
\text { Population }\end{array}$} \\
\hline 3A & 593702925 & S3A_593702925 & $\begin{array}{c}\text { 参考文献 } \\
\text { References }\end{array}$ \\
6A合成六倍体小麦群体 & $\begin{array}{c}\text { A population of synthetic } \\
\text { hexaploid wheat }\end{array}$ \\
6A & 50345873 & S6A_50345873 et al. & \\
6A & 592562315 & S6A_592562315 & \\
7A & 34297426 & S7A_34297426 & \\
1B & 6867825 & S1B_6867825 & \\
2B & 502127437 & S2B_502127437 & \\
3B & 548275272 & S3B_548275272 & \\
3B & 655010350 & S3B_655010350 & \\
6B & 109760004 & S6B_109760004 & \\
6B & 32333184 & S6B_32333184 & \\
6B & 576856920 & S6B_576856920 & \\
6B & 658724336 & S6B_658724336 & \\
2D & 631996199 & S2D_631996199 & \\
3D & 45073985 & S3D_45073985 & \\
\hline
\end{tabular}

${ }^{a}$ 在 2 个环境和 BLUE 值(阈值为 $-\log _{10}(P$-value $\geq 3)$ ) 中均与籽粒钙含量显著关联的标记。

${ }^{\text {a }}$ Consistently significant markers were detected in the two environments and BLUEs values with threshold of $-\log _{10}(P$-value $\geq 3)$.

\section{3 小麦籽粒钙含量根际工程研究}

钙是以钙离子形式从小麦根系进入小麦根部, 然后再转移到地上部分(如种子)。农药和化肥的长期 持续使用, 土壤肥力和稳定性受到严重影响, 最终 导致作物养分利用效率低下 ${ }^{[59]}$ 。根瘤菌和内生菌是 一组重要的农用土壤微生物区系, 根据功能活性分 为生物化肥、植物生长调节物质、植物根际生物修 复剂和生物农药 ${ }^{[59,82]}$ 。根际工程主要是借助生物介 质即促进植物生长的根瘤菌和内生菌等来提高作物 养分利用效率, 其中根瘤菌可直接通过辅助营养同 化(如钲等必需矿物质)或调解作物激素含量, 减少 各种病原菌及其毒素对作物生长发育的影响 ${ }^{[59]}$ 。研 究发现生物接种不同内生菌通过提高土壤肥力、细 胞伸长和改变重金属胁迫下土壤矿物质的生物有效 性来刺激矿物质获取、生物量增加和植物加速修复。

\section{5 问题与展望}

\section{1 籽粒富集钙元素小麦种质篮选与创制}

自绿色革命实现以来, 小麦单产明显增加, 也 导致了必需矿物质营养元素含量大大降低, 即“稀释 效应”, [3]。随着人们对矿物质元素在人类健康中所起 作用的认识不断提高, 相关的生物强化项目在国内 外相继展开。小麦籽粒中含有钙等各种具有重要生 理效应的功能性因子。选育和利用富钙小麦品种是
克服钙元素缺乏最经济有效的途径, 而籽粒富集钙 元素小麦种质资源的䇻选、研究与利用是富钙小麦 育种的重要基础。小麦籽粒钙含量在不同基因型间 存在明显差异, 这为富钙小麦新品种的筛选及选育 提供了广泛的种质资源和空间。现有的大多数高钙 含量小麦种质篮选研究仅在一年或一点开展的, 因 此还需在不同生态区，进行多年多点鉴定试验，篮 选出籽粒钙含量高的小麦品种 (系), 为富钙小麦育 种提供优良的种质资源, 为合理选配亲本提供参 考。同时, 以高钙含量种质材料为基础, 对各地大面 积推广种植的代表性品种进行多种方式的改良, 创 制出中间材料，为富钙小麦新品种培育提供优良的 育种材料。

5.2 常规育种技术与生物技术紧密结合提高小 麦籽粒钙含量

人类所需的营养, 主要依赖于植物性食品, 即 从主粮作物可食部分获取足够的矿物质、蛋白质和 维生素等必不可少的营养素。通过农艺生物强化或 常规育种技术增加主粮作物的矿物质元素含量, 仍 是全球科学家的一项艰巨任务。尽管已取得了一些 成绩, 但在大多数情况下, 仍未达到预期目标。为了使 作物最大程度地吸收养分并将其转移到不同的经济器 官, 科学家们定制并实施了一些生物强化策略, 但在 当前的农业和环境问题上, 这些方法并不十分有效。 
小麦籽粒是最重要的经济器官, 其籽粒钙含量 与小麦产量和品质密切相关, 是品种选育和品质评 估的重要依据之一。通过遗传改良途径进行小麦钙 营养强化, 需要常规育种技术与生物技术紧密结合, 加快育种步伐，提高富钙小麦育种效率。生物技术 主要包括QTL定位分析、分子标记辅助选择和基因 组选择(genomic selection)。其研究思路是在种质资 源篮选的基础上, 利用目标性状的分离群体, 通过 QTL作图鉴定，获得与控制小麦籽粒钙含量QTL紧 密连锁的分子标记, 并对控制籽粒钙含量QTL进行 精细定位和功能分析, 克隆到控制小麦籽粒钙含量 的主效基因，最终培育出抗逆、广适、高产的健康 型小麦新品种。目前，选育并推广种植了锌生物强 化小麦品种Zinc Shakti、Zincol 2016 ${ }^{[11]}$ 和富锌小麦品 种中麦 $175^{[28]}$ 。

\section{3 富钙小麦食品开发利用}

随着生活水平普遍提高和医疗保健意识增强, 人们对作为主粮作物的小麦提出了新要求，其品质 营养状况越来越成为关注的焦点。尽管食物供需稳 定增长，但世界上大部分人口尤其发展中国家人口 仍无法从食物中获得足够的营养，且这些食物总体 营养成分较差 ${ }^{[59]}$ 。因此，选育富钙小麦新品种的同 时，还应改良富钙小麦食品加工工艺和开发富钙小 麦食品。除了铜元素外, 所有矿物质营养元素主要 存在于小麦敖皮和胚芽中，因此生产生物强化面食 需使用全麦粉 ${ }^{83]}$ 。富钙小麦加工成全麦粉或将其全 麦粉与精制面粉配粉制成健康营养食品，以提高钙 元素的生物有效性，这在缺乏膳食纤维的饮食结构 中或贫困地区显得尤为重要。钙含量在小麦籽粒不 同部位存在差异，因对富钙小麦籽粒不同部位分别 进行加工和食品开发是一个重要发展方向。麦胚可 直接工业剥去、压片后制成富钙营养食品。胚芽可 制成矿物质营养元素丰富的胚芽超微粉。麦麸可制 作纤敖食品或直接作为掺粉提高食品中钙含量。

\section{References}

[1] Weaver C M, Peacock M. Calcium. Adv Nutr, 2019, 10: 546-548.

[2] Kumssa D B, Joy E J M, Ander E L, Watts M J, Young S D, Walker S, Broadley M R. Dietary calcium and zinc deficiency risks are decreasing but remain prevalent. $S c i$ Rep, 2015, 5: 10974

[3] Gomez-Coronado F, Almeida A S, Santamaria O, Cakmak I, Poblaciones M J. Potential of advanced breeding lines of bread making wheat to accumulate grain minerals $(\mathrm{Ca}, \mathrm{Fe}, \mathrm{Mg}$ and $\mathrm{Zn}$ ) and low phytates under Mediterranean conditions. J Agron Crop Sci, 2019, 205: 341-352.
[4] 顾景范. 《中国居民营养与慢性病状况报告(2015)》解读. 营 养学报, 2016, 38: 525-529.

Gu J F. Interpretation of "Report on Nutrition and Chronic Diseases of Chinese Residents (2015)". Acta Nutr Sin, 2016, 38: 525-529 (in Chinese).

[5] Bouillon R, Antonio L. Nutritional rickets: historic overview and plan for worldwide eradication. J Steroid Biochem, 2020, 198: 105563.

[6] 邱贵兴. 老年骨质疏松性骨折的治疗策略. 中华老年骨科与康 复电子杂志, 2015, 1(1): 1-5.

Qiu G X. Treatment strategies for osteoporotic fractures in the elderly. Chin J Geriatr Orthop Rehabil (Electronic Edn), 2015, 1(1): 1-5 (in Chinese).

[7] Weaver C M, Heaney R P. Calcium in Human Health. Totowa, New Jersey: Humana Press, 2006. pp 1-3.

[8] Benjakul S, Karnjanapratum S. Characteristics and nutritional value of whole wheat cracker fortified with tuna bone bio-calcium powder. Food Chem, 2018, 259: 181-187.

[9] Bouis H E, Saltzman A. Improving nutrition through biofortification: a review of evidence from harvestplus, 2003 through 2016. Glob Food Secur, 2017, 12: 49-58.

[10] Cheema S A, Rehman H U, Kiran A, Bashir K, Wakeel A. Progress and prospects for micronutrient biofortification in rice/wheat. In: Hossain M A, Kamiya T, Burritt D J, Tran L S P, Fujiwara T, eds. Plant Micronutrient Use Efficiency: Molecular and Genomic Perspectives in Crop Plants. NY, US: Academic Press, 2018. pp 261-278.

[11] Saini D K, Devi P, Kaushik P. Advances in genomic interventions for wheat biofortification: a review. Agronomy-Basel, 2020, 10(1): 62.

[12] Hoekenga O. Genomics of mineral nutrient biofortification: calcium, iron and zinc. In: Tuberosa R, Graner A, Frison E, eds. Genomics of Plant Genetic Resources. Springer Science + Business Media Dordrecht, 2014. pp 431-454.

[13] 张勇, 郝元峰, 张艳, 何心尧, 夏先春, 何中虎. 小麦营养和 健康品质研究进展. 中国农业科学, 2016, 49: 4284-4298.

Zhang Y, Hao Y F, Zhang Y, He X Y, Xia X C, He Z H. Progress in research on genetic improvement of nutrition and health qualities in wheat. Sci Agric Sin, 2016, 49: 4284-4298 (in Chinese with English abstract).

[14] 何中虎, 庄巧生, 程顺和, 于振文, 赵振东, 刘旭. 中国小麦 产业发展与科技进步. 农学学报, 2018, 8(1): 99-106.

He Z H, Zhuang Q S, Cheng S H, Yu Z W, Zhao Z D, Liu X. Wheat production and technology improvement in China. J Agric, 2018, 8(1): 99-106 (in Chinese with English abstract).

[15] 王秀敏, 谢令琴, 刘艳苏, 胡珍荣. 原子吸收光谱法测定小麦 品种子粒中钾钠钙镁的含量. 河北农业大学学报, 2003, 26(4): 90-97.

Wang X M, Xie L Q, Liu Y S, Hu Z R. Determination of the kalium, sodium, calcium and magnesium content in wheat seeds by atomic absorption spectroscopy. J Agric Univ Hebei, 2003, 26(4): 90-97 (in Chinese with English abstract).

[16] 张明艳, 郁一凡, 封超年, 郭文善, 朱新开, 李春燕, 彭永欣. 不同基因型小麦籽粒、面粉和敖皮中 $\mathrm{Ca}$ 和 $\mathrm{Zn}$ 含量的差异. 麦 类作物学报, 2011, 31: 240-245.

Zhang M Y, Yu Y F, Feng C N, Guo W S, Zhu X K, Li C Y, Peng 
Y X. Differences of Calcium and Zinc contents among four, grain and bran of different wheat varieties. $J$ Triticeae Crops, 2011, 31: 240-245 (in Chinese with English abstract).

[17] 刘三才, 李为喜, 张晓芳, 朱志华. 小麦强化营养粉中钙、锌 含量的盐酸浸提快速测定. 麦类作物学报, 2007, 27(1): 63-66. Liu S C, Li W X, Zhang X F, Zhu Z H. Rapid determination of Calcium and Zinc in nutrient enrichment flour of wheat with hydrochloric acid extraction. $J$ Triticeae Crops, 2007, 27(1): 63-66 (in Chinese with English abstract).

[18] 高雅洁, 王朝辉, 王森, 靳静静, 曹寒, 戴健, 于荣. 石灰性土 壤施用氯化钙对冬小麦生长及钙锌吸收的影响. 植物营养与 肥料学报, 2015, 21: 719-726.

Gao Y J, Wang C H, Wang S, Jin J J, Cao H, Dai J, Yu R. Effects of calcium chloride on winter wheat yield and uptake of $\mathrm{Ca}$ and Zn in calcareous soil. J Plant Nutri Fert, 2015, 21: 719-726 (in Chinese with English abstract).

[19] 石荣丽, 邹春琴, 芮玉奎, 张学勇, 夏晓平, 张福锁. ICP-AES 测定中国小麦微核心种质库籽粒矿质养分含量. 光谱学与光 谱分析, 2009, 29: 1104-1107.

Shi R L, Zou C Q, Rui Y K, Zhang X Y, Xia X P, Zhang F S. Application of ICP-AES to detecting nutrients in grain of wheat core collection of China. Spectros Spec Anal, 2009, 29: 1104-1107 (in Chinese with English abstract).

[20] 季英苗. 不同小麦品种(系)中主要矿质元素的含量比较及与品 质的关系. 中国科学院成都生物研究所硕士学位论文, 四川 成都, 2009.

Ji Y M. Study on the Determination and Correlation Analysis with Wheat Quality of the Contents of the Main Mineral Elements in Different Species of Wheat. MS Thesis of Chengdu Institute of Biology, Chinese Academy of Sciences, Chengdu, Sichuan, China, 2009 (in Chinese with English abstract).

[21] Bhatta M, Baenziger P S, Waters B M, Poudel R, Belamkar V, Poland J, Morgounov A. Genome-wide association study reveals novel genomic regions associated with 10 grain minerals in synthetic hexaploid wheat. Int J Mol Sci, 2018, 19: 3237.

[22] 李刚, 郑若锋. $X$ 射线苂光光谱法测定植物样品中 12 种元素含 量. 理化检验: 化学分册, 2012, 48: 1433-1437.

Li G, Zheng N F. XRFS determination of 12 elements in plant samples. Physic Test Chem Anal (Part B: Chem Anal), 2012, 48: 1433-1437 (in Chinese with English abstract).

[23] 李春燕, 封超年, 王亚雷, 张容, 郭文善, 朱新开, 彭永欣. 小 麦籽粒不同部位的矿质元素组成与其含量差异. 植物生理学 报, 2007, 43: 1077-1081.

Li C Y, Feng C N, Wang Y L, Zhang R, Guo W S, Zhu X K, Peng $\mathrm{Y} X$. Differences of mineral elements compositions and their contents among different positions of wheat grains. Plant Physio $J$, 2007, 43: 1077-1081 (in Chinese with English abstract).

[24] 王广西, 胡燕, 罗琼, 李丹, 陈诚, 赖万昌, 翟娟. 波长色散 X 射线苂光光谱法分析小麦籽粒中矿质元素. 分析试验室, 2017, 36: 663-666.

Wang G X, Hu Y, Luo Q, Li D, Chen C, Lai W C, Zhai J. Analysis of mineral elements in wheat grains by wavelength dispersive X-ray fluorescence spectrometry. Chin J Anal Lab, 2017, 36: 663-666 (in Chinese with English abstract).

[25] 田旷达, 邱凯贤, 李祖红, 吕亚琼, 张秋菊, 熊艳梅, 闵顺耕. 近红外光谱法结合最小二乘支持向量机测定烟叶中钙、镁元
素. 光谱学与光谱分析, 2014, 34: 3262-3266.

Tian G D, Qiu K X, Li Z H, Lyu Y Q, Zhang Q J, Xiong Y M, Min S G. Determination of calcium and magnesium in tobacco by near-infrared spectroscopy and least squares-support vector machine. Spectros Spec Anal, 2014, 34: 3262-3266.

[26] 蒋淑丽. 稻米矿质元素分析及其近红外测定技术的研究. 浙江 大学博士学位论文, 浙江杭州, 2007. pp 68-81.

Jiang S L. The Study of Determination and the Calibration Model Optimization by Near Infrared Reflectance Spectroscopy (NIRS) for Mineral Element Contents in Rice (Oryza stativa L.). PhD Dissertation of Zhejiang University, Hangzhou, Zhejiang, China, 2007. pp 68-81 (in Chinese with English abstract).

[27] 王秀敏, 许民安, 常淑惠, 谷俊涛, 谢令琴. 冬小麦品种子粒 钙含量的遗传研究. 河北农业大学学报, 2002, 25(4): 25-28.

Wang X M, Xu M A, Chang S H, Gu J T, Xie L Q. Heredity study on grain calcium content of winter wheat varieties. J Agric Univ Hebei, 2002, 25(4): 25-28 (in Chinese with English abstract).

[28] Zhang Y, Song Q, Yan J, Tang J, Zhao R, Zhang Y, He Z, Zou C, Ortiz-Monasterio I. Mineral element concentrations in grains of Chinese wheat cultivars. Euphytica, 2010, 174: 303-313.

[29] Guttieri M J, Baenziger P S, Frels K, Carver B, Arnall B, Waters B M. Variation for Grain mineral concentration in a diversity panel of current and historical great plains hard winter wheat germplasm. Crop Sci, 2015, 55: 1035-1052.

[30] Alomari D Z, Eggert K, von Wiren N, Pillen K, Roder M S. Genome-wide association study of calcium accumulation in grains of European wheat cultivars. Front Plant Sci, 2017, 8: 1797.

[31] 傅兆麟, 王海燕, 郭孙黎, 宋创业, 张铮. 黄淮麦区主要小麦 种质资源钙含量测定. 淮北煤炭师范学院学报(自然科学 版), 2008, 29(1): 41-44.

Fu Z L, Wang H Y, Guo S L, Song C Y, Zhang Z. The determing report of $\mathrm{Ca}$ content in seed for the main wheat idioplasm resources in Huanghai wheat area. J Huaibei Coal Indus Tech Coll (Nat Sci), 2008, 29(1): 41-44 (in Chinese with English abstract).

[32] 夏瑞雪, 魏帅, 郭波莉, 曹东梅. 豫北地区小麦籽粒矿质元素 含量分析. 核农学报, 2017, 31: 516-523.

Xia R X, Wei S, Guo B L, Cao D M. Analysis of mineral element content of wheat in northern area of Henan province. J Nucl Agric Sci, 2017, 31: 516-523 (in Chinese with English abstract).

[33] 孙宪印, 田枫, 米勇, 牟秋焕, 王瑞霞, 王超, 元晓蕾, 钱兆国, 吴科, 李斯深. 小麦重组自交系群体籽粒主要矿质元素含量 的分析. 麦类作物学报, 2016, 36: 872-877.

Sun X Y, Tian F, Mi Y, Mou Q H, Wang R X, Wang C, Qi X L, Qian Z G, Wu K, Li S S. Analysis of major mineral elements concentration in grain of wheat recombinant inbred lines. $J$ Triticeae Crops, 2016, 36: 872-877 (in Chinese with English abstract).

[34] 王健胜, 吴政卿, 周正富, 马爱锄, 刘军, 泉岳恩, 李文旭, 王 亚欢, 李静婷, 赵干卿, 雷振生. 国内外小麦种质主要矿质元 素含量的评价分析. 分子植物育种, 2018, 16: 7550-7557.

Wang J S, Wu Z Q, Zhou Z F, Ma A C, Liu J, Chao Y E, Li W X, Wang Y O, Li J T, Zhao G Q, Lei Z S. Evaluation and analysis of major mineral elements content in domestic and foreign wheat germplasm. Mol Plant Breed, 2018, 16: 7550-7557 (in Chinese with English abstract). 
[35] Rehman A, Farooq M, Nawaz A, Al-Sadi A M, Al-Hashmi K S, Nadeem F, Ullah A. Characterizing bread wheat genotypes of Pakistani origin for grain zinc biofortification potential. $J$ Sci Food Agric, 2018, 98: 4842-4836.

[36] Akcura M, Kokten K. Variations in grain mineral concentrations of Turkish wheat landraces germplasm. Qual Assur Saf Crop, 2017, 9(2): 1-8.

[37] Hocaolu O, Akcura M, Kaplan M. Changes in the grain element contents of durum wheat varieties of Turkey registered between 1967-2010. Commun Soil Sci Plan, 2020, 51: 431-439.

[38] Balint A F, Kovacs G, Erdei L, Sutka J. Comparison of the Cu, Zn, $\mathrm{Fe}, \mathrm{Ca}$ and $\mathrm{Mg}$ contents of the grains of wild, ancient and cultivated wheat species. Cereal Res Commun, 2001, 29: 375-382.

[39] Calderini D F, Ortiz-Monasterio I. Are synthetic hexaploids a means of increasing grain element concentrations in wheat? Euphytica, 2003, 134: 169-178.

[40] Pongrac P, Arcon I, Castillo-Michel H, Vogel-Mikus K. Mineral element composition in grain of awned and awnletted wheat (Triticum aestivum L.) cultivars: tissue-specific iron speciation and phytate and non-phytate ligand ratio. Plants-Basel, 2020, 9(1): 79.

[41] 胡秋辉, 陈历程, 吴莉莉, 曹延松, 程万和. 黑小麦营养成分 分析及其深加工制品前景展望. 食品科学, 2001，22(12): $50-52$.

Hu Q H, Chen L C, Wu L L, Cao Y S, Cheng W H. Nutritional components analysis of the rye and its prospects for further processed products. Food Sci, 2001, 22(12): 50-52 (in Chinese with English abstract).

[42] Nath M, Roy P, Shukla A, Kumar A. Spatial distribution and accumulation of calcium in different tissues, developing spikes and seeds of finger millet genotype. J Plant Nutr, 2013, 36: $539-550$

[43] Chauhan N, Sankhyan N K, Sharma R P, Singh J, Gourav. Effect of long-term application of inorganic fertilizers, farm yard manure and lime on wheat (Triticum aestivum L.) productivity, quality and nutrient content in an acid alfisol. J Plant Nutr, 2020, 43: 2569-2578.

[44] Galindo F S, Teixeira M C M, Buzetti S, Santini J M K, Boleta E H M, Rodrigues W L. Macronutrient accumulation in wheat crop (Triticum aestivum L.) with Azospirillum brasilense associated with nitrogen doses and sources. J Plant Nutr, 2020, 43: 1057-1069.

[45] Dincsoy M, Sonmez F. The effect of potassium and humic acid applications on yield and nutrient contents of wheat (Triticum aestivum L. var. Delfii) with same soil properties. J Plant Nutr, 2019, 42: 2757-2772.

[46] Popko M, Michalak I, Wilk R, Gramza M, Chojnacka K, Gorecki $\mathrm{H}$. Effect of the new plant growth biostimulants based on amino acids on yield and grain quality of winter wheat. Molecules, 2018, 23: 470 .

[47] 李可懿, 王朝辉, 赵护兵, 赵娜, 高亚军, Lyons G. 黄土高原 旱地小麦与豆科绿肥轮作及施氮对小麦产量和籽粒养分的影 响. 干旱地区农业研究, 2011, 29(2): 110-116.

Li K Y, Wang Z H, Zhao H B, Zhao N, Gao Y J, Lyons G. Effect of rotation with legumes and $\mathrm{N}$ fertilization on yield and grain nutrient contents of wheat in dryland of the Loess Plateau. Agric
Res Arid Areas, 2011, 29(2): 110-116 (in Chinese with English abstract).

[48] Klikocka H, Marks M, Barczak B, Szostak B, Podlesna A, Podlesny J. Response of spring wheat to NPK and S fertilization. The content and uptake of macronutrients and the value of ionic ratios. Open Chem, 2018, 16: 1059-1065.

[49] Pszczolkowska A, Okorski A, Olszewski J, Fordonski G, Krzebietke S, Charenska A. Effects of pre-preceding leguminous crops on yield and chemical composition of winter wheat grain. Plant Soil Environ, 2018, 64: 592-596.

[50] Wanic M, Denert M, Trede K. Effect of forecrops on the yield and quality of common wheat and spelt wheat grain. J Elementol, 2019, 24: 369-383.

[51] Hamner K, Weih M, Eriksson J, Kirchmann H. Influence of nitrogen supply on macro- and micronutrient accumulation during growth of winter wheat. Field Crops Res, 2017, 213: 118-129.

[52] 孙发宇, 李长成, 王安, 李韬. 叶面喷施硒酸钠对不同小麦品 种(系)籽粒硒及其他矿质元素含量的影响. 麦类作物学报, 2017, 37: 559-564.

Sun F Y, Li C C, Wang A, Li T. Effect of sodium selenate application on concentrations of selenium and other minerals in grains of different wheat genotypes. $J$ Triticeae Crops, 2017, 37: 559564 (in Chinese with English abstract).

[53] Pongrac P, Kreft I, Vogel-Mikus K, Regvar M, Germ M, Vavpetic P, Grlj N, Jeromel L, Eichert D, Budic B, Pelicon P. Relevance for food sciences of quantitative spatially resolved element profile investigations in wheat (Triticum aestivum L.) grain. $J R S O c$ Interface, 2013, 10: 20130296.

[54] Zhang P P, Ma G, Wang C, Zhu Y J, Guo T C. Mineral elements bioavailability in milling fractions of wheat grain response to zinc and nitrogen application. Agron J, 2019, 111: 2504-2511.

[55] 索全义, 王俊超, 索凤兰. 小麦开花后不同光合器官对籽粒品 质的影响. 麦类作物学报, 2009, 29: 275-278.

Suo Q Y, Wang J C, Suo F L. Effect of different photosynthesis organs on the quality of wheat grains after anthesis. $J$ Triticeae Crops, 2009, 29: 275-278 (in Chinese with English abstract).

[56] 何红霞. 栽培模式对旱地小麦籽粒产量和养分吸收利用的影 响. 西北农林科技大学硕士学位论文, 陕西杨凌, 2018.

He H X. Wheat Grain Yield and Its Nutrient Update and Utilization Affected by Cultivation Patterns in Dryland. MS Thesis of Northwest A\&F University, Yangling, Shaanxi, China, 2018 (in Chinese with English abstract)

[57] Dolijanovic Z, Nikolic S R, Kovacevic D, Djurdjic S, Miodragovic R, Todorovic M J, Djordjevic J P. Mineral profile of the winter wheat grain: effects of soil tillage systems and nitrogen fertilization. Appl Ecol Env Res, 2019, 17: 11757-11771.

[58] Ryan M H, Derrick J W, Dann P R. Grain mineral concentrations and yield of wheat grown under organic and conventional management. J Sci Food Agric, 2004, 84: 207-216.

[59] Chandra A K, Kumar A, Bharati A, Joshi R, Agrawal A, Kumar S. Microbial-assisted and genomic-assisted breeding: a two way approach for the improvement of nutritional quality traits in agricultural crops. 3 Biotech, 2020, 10(2): 2.

[60] Vreugdenhil D, Aarts M G M, Koornneef M, Nelissen H, Ernst W H O. Natural variation and QTL analysis for cationic mineral content in seeds of Arabidopsis thaliana. Plant Cell Environ, 2004, 
27: 828-839.

[61] Singh U M, Metwal M, Singh M, Taj G, Kumar A. Identification and characterization of calcium transporter gene family in finger millet in relation to grain calcium content. Gene, 2015, 566: 37-46.

[62] 张勇, 王德森, 张艳, 何中虎. 北方冬麦区小麦品种籽粒主要 矿物质元素含量分布及其相关性分析. 中国农业科学, 2007, 40: 1871-1876.

Zhang Y, Wang D S, Sun Y, He Z H. Variation of major mineral elements concentration and their relationships in grain of Chinese wheat. Sci Agric Sin, 2007, 40: 1871-1876 (in Chinese with English abstract).

[63] Shen X, Yuan Y P, Zhang H, Guo Y, Zhao Y, Li S S, Kong F M. The hot QTL locations for potassium, calcium, and magnesium nutrition and agronomic traits at seedling and maturity stages of wheat under different potassium treatments. Genes, 2019, 10: 607.

[64] Ilarslan H, Palmer R G, Horner H T. Calcium oxalate crystals in developing seeds of soybean. Ann Bot, 2001, 88: 243-257.

[65] Rao C K, Annadana S. Nutrient biofortification of staple food crops: technologies, products and prospects. In: Benkeblia $\mathrm{N}$ eds. Phytonutritional Improvement of Crops. UK: John Wiley \& Sons Ltd, 2017. pp 113-118.

[66] Oh B C, Choi W C, Park S, Kim Y O, Oh T K. Biochemical properties and substrate specificities of alkaline and histidine acid phytases. Appl Microbiol Biotechnol, 2004, 63: 362-372.

[67] Narwal R P, Malik R S, Yadak H K. Micronutrients in soils and plants and their impact on animal and human health. In Singh $B$ R, McLaughlin M J, Brevik E C, eds. Nexus of Soils, Plants and Human Health. Germany: Catena Soil Sciences, 2017. pp 64-71.

[68] 王晓曦, 贾爱霞, 于中利. 不同出粉率小麦粉的品质特性及营 养组分研究. 中国粮油学报, 2012, 27(1): 6-9.

Wang X X, Jia A X, Yu Z L. Study on wheat meal quality characteristic and nutritive composition of different flour yield. $J$ Chin Cereals Oils Assoc, 2012, 27(1): 6-9 (in Chinese with English abstract).

[69] 高森森, 关二旗, 李萌萌, 市科. 不同出粉率小麦粉矿物质含 量及其加工品质研究. 食品工业, 2018, 39(4): 217-220.

Gao S S, Guan E Q, Li M M, Bian K. Study on mineral content and processing quality of wheat flour with different flour yields. Food Ind, 2018, 39(4): 217-220 (in Chinese with English abstract).

[70] 姬飞腾, 李楠, 邓馨. 喀斯特地区植物钙含量特征与高钙适应 方式分析. 植物生态学报, 2009, 33: 926-935.

Ji F T, Li N, Deng X. Calcium contents and high calcium adaptation of plants in karst areas of China. Chin J Plant Ecol, 2009, 33: 926-935 (in Chinese with English abstract).

[71] Grusak M A, DellaPenna D. Improving the nutrient composition of plants to enhance human nutrition and health. Annu Rev Plant
Phys, 1999, 50: 133-161.

[72] 李鹏, 张兆沛, 有飞燕, 高晓凯, 张联合. 小麦灌浆期籽粒累积 钙的生理特性研究. 山东农业科学, 2017, 49(8): 30-32.

Li P, Zhang Z P, Yu F Y, Gao X K, Zhang L H. Study on physiological characteristics of calcium accumulation in wheat grains at filling stage. Shandong Agric Sci, 2017, 49(8): 30-32 (in Chinese with English abstract).

[73] Peleg Z, Cakmak I, Ozturk L, Yazici A, Jun Y, Budak H, Korol A B, Fahima T, Saranga Y. Quantitative trait loci conferring grain mineral nutrient concentrations in durum wheat $\times$ wild emmer wheat RIL population. Theor Appl Genet, 2009, 119: 353-369.

[74] Shi R L, Tong Y P, Jing R L, Zhang F S, Zou C Q. Characterization of quantitative trait loci for grain minerals in hexaploid wheat (Triticum aestivum L.). J Integr Agric, 2013, 12: 1512-1521.

[75] Morris J, Hawthorne K M, Hotze T, Abrams S A, Hirschi K D. Nutritional impact of elevated calcium transport activity in carrots. Proc Natl Acad Sci USA, 2008, 105: 1431-1435.

[76] Li A L, Zhu Y F, Tan X M, Wang X, Wei B, Guo H Z, Zhang Z L, Chen X B, Zhao G Y, Kong X Y, Jia J Z, Mao L. Evolutionary and functional study of the CDPK gene family in wheat (Triticum aestivum L.). Plant Mol Biol, 2008, 66, 429-443.

[77] Nirgude M, Babu B K, Shambhavi Y, Singh U M, Upadhyaya H D, Kumar A. Development and molecular characterization of genic molecular markers for grain protein and calcium content in finger millet (Eleusine coracana (L.) Gaertn.). Mol Biol Rep, 2014, 41: 1189-1200.

[78] Wattoo J I, Liaqat S, Mubeen H, Ashfaq M, Shahid M N, Farooq A, Sajjad M, Arif M. Genetic mapping of grain nutritional profile in rice using basmati derived segregating population revealed by SSRs. Int J Agric Biol, 2019, 21: 929-935.

[79] Punshon T, Hirschi K, Yang J, Lanzirotti A, Lai B, Guerinot M L. The role of $C A X 1$ and $C A X 3$ in elemental distribution and abundance in Arabidopsis seed. Plant Physiol, 2012, 158: 352-362.

[80] Kokane S B, Pathak R K, Singh M, Kumar A. The role of tripartite interaction of calcium sensors and transporters in the accumulation of calcium in finger millet grain. Biol Plant, 2018, 62: 325-334.

[81] Tabbita F, Pearce S, Barneix A J. Breeding for increased grain protein and micronutrient content in wheat: ten years of the GPC-B1 gene. J Cereal Sci, 2017, 73: 183-191.

[82] Ma Y, Rajkumar M, Zhang C, Freitas F. Beneficial role of bacterial endophytes in heavy metal phytoremediation. J Environ Manage, 2016, 174: 14-25.

[83] Pataco I M, Lidon F C, Ramos I, Oliveira K, Guerra M, Pessoa M F, Carvalho M L, Ramalho J C, Leitao A E, Santos J P, Campos P S, Silva M M, Pais I P, Reboredo F H. Biofortification of durum wheat (Triticum turgidum L. ssp. durum (Desf.) Husnot) grains with nutrients. J Plant Interact, 2017, 12(1): 39-50. 